\title{
Comparison of eye measurements between young Korean women with inborn double eyelids and those with single eyelids
}

\author{
Han Byul Jung, Dong Gil Han, \\ Jeong Su Shim, Yong Jig Lee, \\ Sung-Eun Kim
}

Department of Plastic and Reconstructive Surgery, Catholic University of Daegu School of Medicine, Daegu, Korea
Background Eye measurements in Koreans have been studied extensively, but researchers have reported widely differing values, even for the same parameters. These discrepancies are likely due to inconsistencies in the proportions of subjects with double eyelids included in their studies. We retrospectively studied eye measurements according to the presence or absence of double eyelids, and compared the results to those of previous studies.

Methods We conducted anthropometric measurements of eyes in young (20-29 years) Korean women with no congenital malformation, trauma, or surgery of the eyelids. The participants were dichotomized according to the presence of double eyelids, resulting in 116 eyes with single eyelids and 124 with double eyelids. We measured the palpebral fissure length ( $\mathrm{PFL}$ ), palpebral fissure height (PFH), margin reflex distance 1 (MRD1), intercanthal distance (ICD), interpupillary distance (IPD), outer canthal distance $(O C D)$, and inclination of the palpebral fissure in each eye.

Results The mean PFL was $24.1 \mathrm{~mm}$ in the single-eyelid group and $25.5 \mathrm{~mm}$ in the double-eyelid group. The other mean values for the two groups were as follows: PFH, 8.0 $\mathrm{mm}$ and $9.9 \mathrm{~mm}$, respectively; MRD1, $2.8 \mathrm{~mm}$ and $4.1 \mathrm{~mm}$, respectively; IPD, $61.6 \mathrm{~mm}$ and $62.8 \mathrm{~mm}$, respectively; and $O C D, 85.3 \mathrm{~mm}$ and $87.9 \mathrm{~mm}$, respectively.

Conclusions In the double-eyelid group, the horizontal and vertical length of the eye was greater than in the single-eyelid group, and the mean values of IPD, OCD, PFL, PFH, and MRD1 were statistically significantly higher.

Keywords Anthropometry / Eyelids / Korea

\section{INTRODUCTION}

Anthropometric studies of the eye are conducted in anthropology and in medicine, and provide useful information for medical diag-

Received: Nov 22, 2019 Revised: Dec 27, 2019 Accepted: Dec 30, 2019 Correspondence: Dong Gil Han Department of Plastic and Reconstructive Surgery, Catholic University of Daegu School of Medicine, 33 Duryugongwon-ro 17-gil, Nam-gu, Daegu 42472, Korea Tel: +82-53-650-4578, Fax: +82-53-650-4584, E-mail: dghan1001@cu.ac.kr

Copyright @ 2020 The Korean Society for Aesthetic Plastic Surgery.

This is an Open Access article distributed under the terms of the Creative Commons Attribution Non-Commercial License (https://creativecommons.org/licenses/by-nc/4.0/) which permits unrestricted non-commercial use, distribution, and reproduction in any medium, provided the original work is properly cited. www.e-aaps.org noses and treatment. Many methods are used in anthropometric studies, including actual measurements, photographic measurements, and radiographic measurements. However, photographic measurements are most often performed for the eye because of the simplicity and high repeatability of this method [1]. Studies of eye measurements in Koreans have reported inconsistent values, depending on the researchers, and those studies included different proportions of participants with double eyelids, ranging from $31.7 \%$ to $54.0 \%$ [1-7]. Several studies did not even mention whether subjects with double eyelids were included in their studies [8-11]. In these previous studies, the results varied by up to $20 \%$, even for measurements of the same parameters. As an example, for palpebral fissure height (PFH), Park et al. [8] reported a mean value of 8 $\mathrm{mm}$, compared to $10 \mathrm{~mm}$ for Song et al. [6]. Unlike Westerners, 
many Koreans have single eyelids, but several previous studies did not indicate the distribution of subjects according to the presence or absence of double eyelids. We suspected that the discrepancies in previous results may have been caused by differences in the proportion of subjects with double eyelids who were included in the studies. Therefore, in this study, we performed eye measurements on participants with and without double eyelids, and compared the values with those from other studies through a statistical analysis.

\section{METHODS}

\section{Study participants}

Korean women in their 20s attending medical and nursing colleges were screened for inclusion in our study. Those with congenital malformations, trauma, or surgery of the eyelids were excluded. In total, 240 eyes from 120 participants, including 62 with double eyelids and 58 with single eyelids, were included. The study received approval from the bioethics committee (protocol: IRB CR-19-038). This study conformed to the ethical principles for medical research presented in the Declaration of Helsinki. The participants were informed about the purpose of our study, and signed informed consent forms were collected from them.

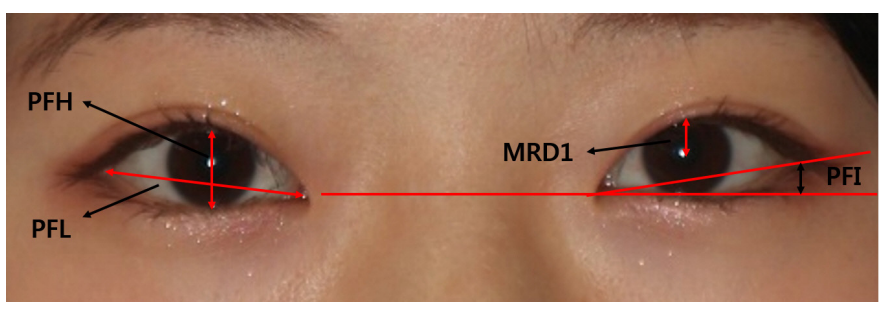

Fig. 1. Eye measurements. PFH, palpebral fissure height ldistance between the inferior margin of the eyelid and the superior margin of the eyelid over the pupil); PFL, palpebral fissure length (distance between the medial and lateral canthus); PFI, palpebral fissure inclination in degrees langle between the intersection of the horizontal reference line with a line drawn through the medial canthus to the lateral canthus); MRD1, margin reflex distance 1 (distance between the pupil center and the superior margin of the eyelid).

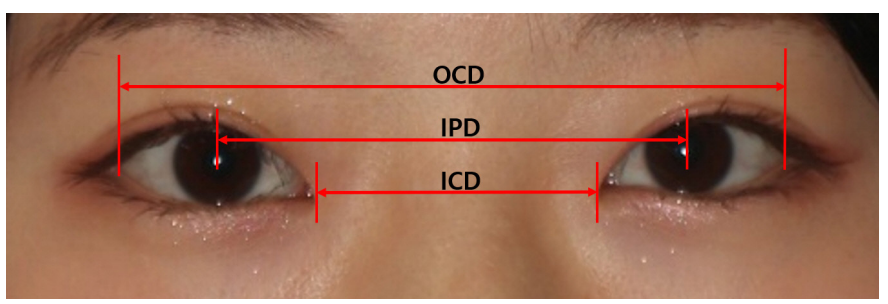

Fig. 2. Canthal and interpupillary distance measurements. $O C D$, outer canthal distance (distance between the lateral canthi); IPD, interpupillary distance (distance between the pupil centers); ICD, intercanthal distance (distance between the medial canthi).

\section{Anthropometry and photographic analysis}

Frontal-view photographs were taken of all participants using a Nikon D80 digital camera (Nikon Corporation, Tokyo, Japan) with a standard lens. Participants sat $1.0 \mathrm{~m}$ from the camera-representing the standard focal length-with their eyes gazing forward.

We measured the palpebral fissure length (PFL), palpebral fissure height $(\mathrm{PFH})$, margin reflex distance 1 (MRD1), intercanthal distance (ICD), interpupillary distance (IPD), outer canthal distance (OCD), and palpebral fissure inclination (PFI) in each eye. These values were assessed quantitatively using Adobe Photoshop 7.0 (Adobe Inc., San Jose, CA, USA) and a Java-based image processing program (Image J 1.40; National Institutes of Health, Bethesda, MD, USA) [12]. The following measurements were made using standardized photographs: (1) eye measurements-PFL, PFH, PFI, and MRD1 (Fig. 1); (2) canthal and IPD measurements-OCD, IPD, and ICD (Fig. 2).

To minimize potential error due to differences in size among the photographs, the corneal diameter of participants was measured and corrected to match the average corneal diameter $(11.3 \mathrm{~mm})$ of young Korean women [1].

\section{Statistical analysis}

Statistical analysis was performed using SPSS version 19.0 (IBM Corp., Armonk, NY, USA). The values of the single-eyelid group and the double-eyelid group were compared using the independent t-test. P-values less than 0.05 were considered to indicate statistical significance.

Table 1. Comparison of objective measurements in the double-eyelid and single-eyelid groups

\begin{tabular}{lccc}
\hline Objective outcome & Double eyelids & Single eyelids & P-value \\
\hline PFL (mm) & $25.5 \pm 1.7$ & $24.1 \pm 1.5$ & $<0.001$ \\
PFH (mm) & $9.9 \pm 1.0$ & $8.0 \pm 0.9$ & $<0.001$ \\
PFI ( $\left.{ }^{\circ}\right)$ & $8.4 \pm 2.9$ & $8.8 \pm 3.0$ & 0.275 \\
MRD1 (mm) & $4.1 \pm 0.7$ & $2.8 \pm 0.8$ & $<0.001$ \\
OCD (mm) & $87.9 \pm 4.9$ & $85.3 \pm 3.8$ & $<0.001$ \\
IPD (mm) & $62.8 \pm 3.6$ & $61.6 \pm 3.1$ & 0.012 \\
ICD (mm) & $38.0 \pm 3.1$ & $38.2 \pm 1.8$ & 0.773
\end{tabular}

Values are presented as mean \pm SD.

$\mathrm{PFL}$, palpebral fissure length (distance between the medial and lateral canthus); PFH, palpebral fissure height (distance between the inferior margin of the eyelid and the superior margin of the eyelid over the pupil); PFI, palpebral fissure inclination in degrees langle between the intersection of the horizontal reference line with a line drawn through the medial canthus to the lateral canthus); MRD1, margin reflex distance 1 (distance between the pupil center and the superior margin of the eyelid); OCD, outer canthal distance (distance between the lateral canthi); IPD, interpupillary distance (distance between the pupil centers); ICD, intercanthal distance (distance between the medial canthi). 


\section{RESULTS}

The mean PFL was $24.1 \pm 1.5 \mathrm{~mm}$ in the single-eyelid group and $25.5 \pm 1.7 \mathrm{~mm}$ in the double-eyelid group. The PFH of the two groups was $8.0 \pm 0.9 \mathrm{~mm}$ and $9.9 \pm 1.0 \mathrm{~mm}$, respectively, and the MRD1 was $2.8 \pm 0.8 \mathrm{~mm}$ and $4.1 \pm 0.7 \mathrm{~mm}$, respectively. The ICD was $38.2 \pm 1.8 \mathrm{~mm}$ and $38.0 \pm 3.1 \mathrm{~mm}$, the IPD was $61.6 \pm 3.1 \mathrm{~mm}$ and $62.8 \pm 3.6 \mathrm{~mm}$, the $\mathrm{OCD}$ was $85.3 \pm 3.8 \mathrm{~mm}$ and $87.9 \pm 4.9 \mathrm{~mm}$, and the PFI was $8.8^{\circ} \pm 3.0^{\circ}$ and $8.4^{\circ} \pm 2.9^{\circ}$ in the two groups, respectively (Table 1).

\section{DISCUSSION}

Various methods of making eyelid measurements exist, but photogrammetry requires minimal time and enables the measurements to be repeated; therefore, this method has been used in many studies of eye measurements in Koreans.

However, the results of some studies varied widely, leading us to consider that distribution of participants in those studies according to the presence or absence of double eyelids may have caused these disparities. The mechanism of eyelid enlargement after double eyelidplasty involves differences in tissue thickness, the lower positioning of the orbit septum, and the prominent preaponeurotic fat resulting in a single full lid. In other words, a dynamic connection of the levator aponeurosis to the tarsus and skin makes upper-eyelid excursion easier in subject with double eyelids [13]. Similarly, we hypothesized that there may be differences in eye size between people with inborn double eyelids and those with single eyelids.

Western research has included mostly participants with double eyelids, but many Koreans have single eyelids. Some studies have considered the proportion of double to single eyelids in their participants, but others have not mentioned this issue.

In this study, we dichotomized participants according to whether they had single or double eyelids, and measured seven parameters (PFL, PFH, PFI, MRD1, ICD, IPD, and OCD), which were then compared between the groups. The accuracy of the proportional measurement program was verified by comparing differences between measured values and values from the proportional measurement program [14]. Previous studies have shown that the cor-

Table 2. Differences between the double-eyelid and single-eyelid groups for selected parameters

\begin{tabular}{cccccc}
\hline & PFL & IPD & OCD & $\begin{array}{c}\text { Length from } \\
\text { the medial } \\
\text { canthus to the } \\
\text { pupil center }\end{array}$ & $\begin{array}{c}\text { Length from } \\
\text { the pupil } \\
\text { center to the } \\
\text { lateral canthus }\end{array}$ \\
\hline Mean $(\mathrm{mm})$ & 1.4 & 1.2 & 2.6 & 0.5 & 0.7
\end{tabular}

PFL, palpebral fissure length (distance between the medial and lateral canthus); IPD, interpupillary distance (distance between the pupil centers); OCD, outer canthal distance (distance between the lateral canthi). neal diameter is slightly different between men and women, but there is no significant difference by age [1]. Therefore, adjusted the eye measurements using a standard corneal diameter of $11.3 \mathrm{~mm}$.

The proportional measurements using digital photography were made using Adobe Photoshop (Adobe Inc.) and a Java-based image processing program (Image J 1.40). Many parameters were measured at the same time, and the measurements could be repeated at any time [12]. The mean values of PFL, PFH, MRD1, IPD, and OCD for the single-eyelid group showed statistically significant differences compared to the double-eyelid group (Table 1). The following between-group differences were observed: PFL, $1.4 \mathrm{~mm}$; IPD, $1.2 \mathrm{~mm}$; OCD, $2.6 \mathrm{~mm}$, distance from the medial canthus to the pupil center, $0.5 \mathrm{~mm}$; and distance from the pupil center to the lateral canthus, $0.7 \mathrm{~mm}$ (Table 2).

The horizontal and vertical lengths of the eyes were compared with the findings of previous studies. For PFL, Cho et al. [9] reported a mean value of $35.5 \mathrm{~mm}$, while Song et al. [6] reported a value of $24.6 \mathrm{~mm}$. In this study, the mean value of PFL was $24.1 \mathrm{~mm}$ in the single-eyelid group and $25.5 \mathrm{~mm}$ in the double-eyelid group. For IPD, Song et al. [3] reported a mean value of $64.3 \mathrm{~mm}$. Hwang et al. [10] reported that the mean value of IPD was $66.6 \mathrm{~mm}$. In this study, the mean value of IPD was $61.6 \mathrm{~mm}$ in the group with single eyelids and $62.8 \mathrm{~mm}$ in the group with double eyelids. For OCD, Bae et al. [1] reported a mean value of $87.4 \mathrm{~mm}$, and Song et al. [6] reported a value of $90 \mathrm{~mm}$. In this study, the mean value of OCD was $85.3 \mathrm{~mm}$ in the single-eyelid group and $87.9 \mathrm{~mm}$ in the

Table 3. Eye measurements in previous studies

\begin{tabular}{lcccccc}
\hline \multirow{2}{*}{ Study lyear) } & \begin{tabular}{c} 
Prevalence \\
of double \\
\cline { 5 - 7 }
\end{tabular} & \multicolumn{5}{c}{ Mean (mm) } \\
\cline { 3 - 7 } & eyelid (\%) & PFL & PFH & MRD1 & IPD & OCD \\
\hline Bae et al., 2007 [1] & 44.9 & 27.4 & 8.2 & - & - & 87.4 \\
Kim et al., 1985 [2] & 40 & - & 8.1 & - & - & - \\
Song et al., 1999 [3] & 35.1 & 27.9 & 8.5 & - & 64.3 & - \\
Park et al., 2000 [4] & 54 & 28 & 9.1 & - & - & - \\
Song et al., 2001 [5] & 31.7 & 27.8 & 9.1 & 2.7 & - & - \\
Song et al., 2002 [6] & 47.2 & 24.6 & 10 & - & - & 90 \\
Park et al., 2008 [7] & 41.3 & 26.8 & 8.2 & - & - & - \\
Park et al., 1990 [8] & - & 28.4 & 8 & 2.7 & 65.1 & - \\
Cho et al., 1993 [9] & - & 35.5 & 8.5 & - & - & 99.7 \\
Hwang et al., 1996 [10] & - & - & - & - & 66.6 & 100 \\
Seo et al., 2009 [11] & - & - & 8.2 & 3.1 & - & - \\
Our study (single eyelid) & - & 24.1 & 8.0 & 2.8 & 61.6 & 85.3 \\
Our study (double eyelid] & - & 25.5 & 9.9 & 4.1 & 62.8 & 87.9 \\
\hline
\end{tabular}

PFL, palpebral fissure length (distance between the medial and lateral canthus); PFH, palpebral fissure height (distance between the inferior margin of the eyelid and the superior margin of the eyelid over the pupil); MRD1, margin reflex distance 1 (distance between the pupil center and the superior margin of the eyelid); IPD, interpupillary distance (distance between the pupil centers); OCD, outer canthal distance (distance between the lateral canthi). 
double-eyelid group.

For PFH, Park et al. [8] reported a mean value of $8 \mathrm{~mm}$, compared to the value of $10 \mathrm{~mm}$ reported by Song et al. [6]. In this study, the mean value of PFH was $8.0 \mathrm{~mm}$ in the single-eyelid group and $9.9 \mathrm{~mm}$ in the double-eyelid group. For MRD1, Seo and Ahn [11] reported a mean value of $3.1 \mathrm{~mm}$, while Song et al. [5] reported a value of $2.7 \mathrm{~mm}$. In this study, the mean value of MRD1 was 2.8 $\mathrm{mm}$ in the group with single eyelids and $4.1 \mathrm{~mm}$ in the group with double eyelids.

As mentioned above, the results of eye measurements varied across each study. In this study, the parameters that showed statistically significant differences according to the presence or absence of double eyelids were compared with previous studies. The proportion of participants with double eyelids in each study is also provided (Table 3).

Our results showed that the distance between the medial canthi (ICD) and the inclination between the medial canthus and the lateral canthus (PFI) did not show a statistically significant difference between the two groups, but the measurements were higher in the double-eyelid group than in the single-eyelid group. An illustration was made to reflect this finding, showing that the dimensions of the eye were larger in the double-eyelid group (Fig. 3). The length measurements in the four directions around the pupil center were larger in the double-eyelid group, and accordingly, the mean values of IPD, OCD, PFL, PFH, and MRD1 were statistically significantly

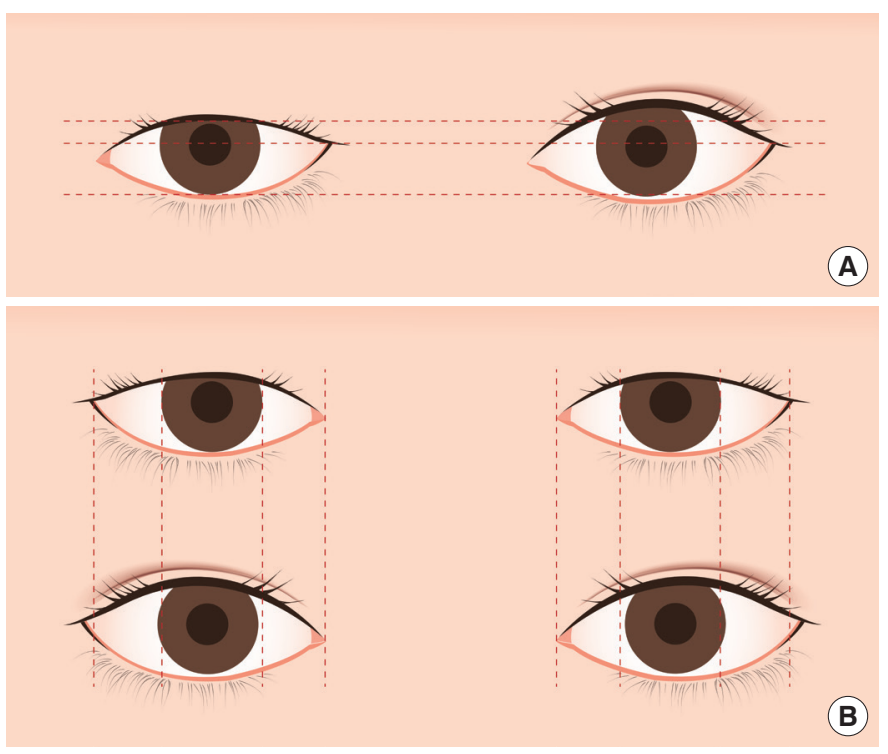

Fig. 3. Differences between the measurements of eyes without and with inborn double eyelids. An illustration was created to reflect our results. (A) In eyes with double eyelids, the vertical length of the eye is greater. The upper and lower lengths from the pupil center appear longer. (B) In eyes with double eyelids, the horizontal length of the eye is also greater. The medial and lateral lengths from the pupil center appear longer. larger in the double-eyelid group.

A number of previous studies have compared preoperative and postoperative measurements in patients undergoing eyelid surgery, with significant results $[12,15,16]$. However, no studies have compared the measurements of individuals with double eyelids who have not undergone surgery to those of individuals with single eyelid. In this study, eye measurements in Koreans were analyzed according to the presence or absence of double eyelids. Statistically significant differences were obtained for some parameters, which could be meaningful data for subsequent eyelid surgery or for Korean anthropometric studies.

Furthermore, the results of our study may be related to the reasons why many people without double eyelids in Korea undergo double-eyelid surgery (medial and/or lateral epicanthoplasty) to enlarge their eyes.

However, our study had some limitations. First, the number of participants was relatively small, meaning that our measurements may not be fully representative of young adults in Korea. Therefore, a further study is needed with a larger number of participants with and without double eyelids. Second, in other studies, the average corneal diameter was found to be 11-13 mm $[1,3,8]$. Based on these results, we standardized measurements based on an average corneal diameter of $11.3 \mathrm{~mm}$ because that average value was obtained from a large sample (827 people) of people from a similar age group (19-27 years). Nevertheless, it is necessary to consider the possibility of error introduced by using an estimated corneal diameter for standardization.

\section{NOTES}

\section{Conflict of interest}

No potential conflict of interest relevant to this article was reported.

\section{Ethical approval}

The study was approved by the Institutional Review Board of Daegu Catholic University Medical Center (IRB No. CR-19-038) and performed in accordance with the principles of the Declaration of Helsinki. The participants provided written informed consent for the publication and the use of their images.

\section{ORCID}

Han Byul Jung

Dong Gil Han Jeong Su Shim Yong Jig Lee Sung-Eun Kim

\section{REFERENCES}

1. Bae TH, Kim TC, Kim WS, et al. A photogrammetic study of the eyes 
in Korean youths. J Korean Soc Plast Reconstr Surg 2007;34:37-43.

2. Kim YH, Kim YS, Lee SI. A stastical study of upper eyelids of Korean young women. J Korean Soc Plast Reconstr Surg 1985;12:325-9.

3. Song CH, Ahn KY, Han DC, et al. The age-related anthropometric analysis of eyelids and orbits in Koreans. J Korean Soc Plast Reconstr Surg 1999;109:1131-7.

4. Park JW, Lee BH, Jeong SK, et al. Morphological evaluation of upper eyelid in Korean. J Korean Ophthalmol Soc 2000;41:879-85.

5. Song WS, Kim YH, Lee SJ. Morphologic study of upper eyelid contour and functional evaluation of levator palpebrae superioris muscle in adult and young people. J Korean Ophthalmol Soc 2001;42:1523-9.

6. Song WC, Park SH, Koh KS. Metric and non-metric characteristics of Korean eyes using satadardized photographs. Korean J Phys Anthropol 2002;15:95-107.

7. Park DH, Choi WS, Yoon SH, et al. Anthropometry of Asian eyelids by age. Plast Reconstr Surg 2008;121:1405-13.

8. Park DM, Song JW, Han KH, et al. Anthropometry of Korean eyelids. J Korean Soc Plast Reconstr Surg 1990;17:822-41.

9. Cho JH, Han KW, Kang JS. Normal anthropometric values and standardized templates of Korean face and head. J Korean Soc Plast Re- constr Surg 1993;20:995-1005.

10. Hwang K, Ough MH, Baik SH. Morphometrical study of interocular distances in Korean adults. J Korean Soc Plast Reconstr Surg 1996;23: 914-20.

11. Seo HR, Ahn HB. Morphological changes of the eyelid according to age. J Korean Ophthalmol Soc 2009;50:1461-7.

12. Park KS, Park DD. Objective outcome measurement after upper blepharoplasty: an analysis of different operative techniques. Aesthetic Plast Surg 2017;41:64-72.

13. Boo-Chai K. Plastic construction of the superior palpebral fold. Plast Reconstr Surg 1963;31:74-8.

14. Kim WS, Hong JS, Kim HK, et al. Photogrammetric study of lip in young population in Korean. J Korean Soc Plast Reconstr Surg 2005; 32:155-60.

15. Kim WJ, Park DH, Han DG. Ten years of results of modified frontalis muscle transfer for the correction of blepharoptosis. Arch Plast Surg 2016;43:172-80.

16. Yeo SH, Han DG. Measurement of the exposed eyeball area and vertical dimension of palpebral fissure after double eyelidplasty. Arch Aesthetic Plast Surg 2017;23:24-9. 